Meta

Journal des traducteurs

Translators' Journal

\title{
La notion de notion en terminologie
}

\section{Allal Assal}

Volume 39, numéro 3, septembre 1994

URI : https://id.erudit.org/iderudit/001912ar

DOI : https://doi.org/10.7202/001912ar

Aller au sommaire du numéro

Éditeur(s)

Les Presses de l'Université de Montréal

ISSN

0026-0452 (imprimé)

1492-1421 (numérique)

Découvrir la revue

Citer cet article

Assal, A. (1994). La notion de notion en terminologie. Meta, 39(3), 460-464.

https://doi.org/10.7202/001912ar d'utilisation que vous pouvez consulter en ligne.

https://apropos.erudit.org/fr/usagers/politique-dutilisation/ 


\title{
LA NOTION DE NOTION EN TERMINOLOGIE
}

\author{
Allal ASSAL \\ Groupe de recherche en terminologie, URA CNRS 1164 , \\ Université de Rouen, Rouen, France
}

Pour les terminologues s'inscrivant dans le cadre de la terminologie classique, la question de la notion semble être résolue par les décisions de l'ISO et leur consécration par les différents organismes nationaux de normalisation. Toutefois, l'examen, même rapide, des différentes définitions produites au cours des vingt dernières années révèle l'indétermination de la notion de notion.

Prenons en considération quelques-unes de ces définitions:

«Construction mentale utilisée pour classer les objets individuels du monde extérieur ou intérieur par une abstraction plus ou moins arbitraire.» (ISO, Recommandation R 704, avril 1968) (cf. Rey 1979:30).

- «Unité mentale produite par l'agrégation d'objets individuels liés par des caractères communs.» (Projet des normes allemandes DIN) (cf. Rey 1979: 30).

- «Ensemble cohérent de jugements sur un objet dont le noyau est constitué par les jugements reflétant les caractères inhérents de l'objet.» (Révision russe de la recommandation ISO, 1977) (cf. Rey 1979: 30).

- «C'est une construction mentale qui représente un objet individuel matériel ou immatériel. La notion consiste en un ensemble de caractères que nous reconnaissons comme étant communs à un certains nombre d'objets individuels et que nous utilisons comme moyen de classification mentale et de communication. Les caractères sont eux aussi des notions.» (Felber 1987: 86).

- «Unité de pensée constituée par abstraction à partir des propriété communes à un ensemble d'objets. Les notions ne sont pas liées aux langues individuelles.» (Norme internationale ISO 1087, $1990: 1$ ).

Comme on peut facilement le constater, la tendance mentaliste de ces définitions est ouvertement déclarée. Nous sommes pleinement d'accord avec A. Rey $(1979: 32)$ pour critiquer vigoureusement cette tendance selon laquelle «les concepts permettent d'appréhender les propriétés caractéristiques objectives qui font qu'une chose est ce qu'elle est» et qui accrédite «l'illusion de concepts correspondant à une réalité extra-humaine, extrasociale, extra-historique, auxquels on accrocherait tel ou tel signe selon les langues».

En outre, ces définitions s'accordent pour considérer la notion comme un produit résultant du regroupement des caractères communs à une classe d'objets individuels. Cette vision concernant avant tout des notions techniques référant à des objets concrets (des appareils, p. ex.), ne peut en aucun cas être généralisée aux notions théoriques abstraites et à certains types de notions qu'on peut qualifier de technologiques.

Voyons d'abord la question des notions technologiques. Ces dernières appartiennent à des disciplines ou à des pratiques qui sont essentiellement des applications de théories scientifiques (c'est le cas, par exemple, du génie enzymatique et des différentes pratiques biotechnologiques). Elles présentent la particularité d'être chargées de théorie. En outre, elles se distinguent des notions purement techniques par le fait qu'elles ne renvoient pas à 
des objets concrets, matériels, observables directement dans la réalité physique, mais à des principes, à des procédés ou à des méthodes déterminées théoriquement et dont on peut observer le déroulement et/ou le résultat; c'est le cas de notions telles que : différenciation, maturation moléculaire et recombinaison génétique, que nous citons ici à titre d'exemple.

Différenciation: «Suite de processus biochimiques programmés génétiquement conférant à une cellule immature toutes les caractéristiques cytologiques et biochimiques nécessaires à un rôle spécialisé autre que la croissance. La cellule différencié est dite mature» (Chopplet 1987: 74).

Maturation moléculaire : «Ensemble des événements biochimiques qui permettent de passer d'un précurseur à une molécule active finale» (Dictionnaire des néologismes officiels 1990: 12).

Recombinaison génétique : «Phénomène conduisant à l'apparition, dans une cellule ou dans un individu, de gènes ou de caractères héréditaires dans une association différente de celle observée chez les cellules ou individus parentaux» (Dictionnaire des néologismes officiels 1990: 15).

Comme on peut le constater, de telles notions, que nous avons appelées technologiques, envisagées du point de vue de la référence, se distinguent nettement des notions techniques à partir desquelles ont été formulées les différentes définitions citées précédemment et qui, firlalement, restent dans 1'horizon de réflexion de beaucoup de terminologues d'inspiration wiistérienne.

De la même façon que ces notions technologiques se distinguent des notions techniques, comme nous venons de le dire, elles se distinguent également des notions théoriques abstraites comme celles qu'on trouve en philosophie, en logique, en mathématique ou en physique, par exemple. Ces dernières, les notions théoriques, ont des référents qui n'existe pas réellement dans le monde physique: ils ne sont pas observables directement en tant qu'objets concrets (comme c'est le cas avec les notions techniques) ou en tant qu'ensemble de processus observables sous un aspect ou un autre (le déroulement ou le résultat) comme c'est le cas avec les notions technologiques. En outre, leur vérité est hypothétique dans le cadre d'une théorie. Cette vérité dépend de la valeur épistémique de la théorie dans laquelle elles interviennent (exemple: les notions de l'infiniment petit et de l'infiniment grand en physique).

De ce qui précède, on peut se rendre compte de l'inadéquation de la définition de la notion en terminologie classique. En effet, devant la diversité des notions (nous en avons explicité trois types, mais il y en a peut être d'autres), on ne peut pas parler de la notion comme si c'était une réalité homogène, comme si c'était quelque chose d'identique partout dans les différents champs du savoir.

Jusqu'ici, nous n'avons fait que montrer l'incapacité classificatoire et descriptive de la définition chosiste et consensuelle de la notion, peut-être d'une manière un peu rapide, mais suffisante pour justifier la nécessité d'une redéfinition.

Pour commencer cet effort définitoire, nous allons considérer les deux termes notion et concept comme synonymes. Cette décision d'établir une équivalence entre les deux termes a été prise par l'ISO et les différentes institutions nationales de normalisation. Pour notre part, nous ne faisons que nous ranger à cette décision qui est peut être artitraire mais très utile, car simplificatrice.

Mais il faut évoquer un autre aspect plus problématique et qu'on ne peut aucunement passer sous silence : la question du rapport entre notion (concept) et signifié. Faut-il distinguer le signifié de la notion, ou au contraire aligner l'un sur l'autre? 
En se basant sur le fait que le signifié prend sa valeur (son sens) à l'intérieur du système de la langue, là où la notion prend la sienne dans le cadre d'une pratique sociale donnée, on serait tenté d'établir d'emblée une distinction nette entre notions et signifiés linguistiques. Mais, pour que cette distinction soit tout à fait juste, il faudra prouver l'indépendance des notions vis-à-vis de la langue. Autrement dit, montrer que la langue n'a aucun impact sur la formation des notions, ce qui est, pensons-nous, au fondement de la pensée d'Eugen Wüster et de ses continuateurs ( $c f$. Felber 1987).

$\mathrm{Si}$ on admet que la langue en tant que système ne conditionne pas les notions et que celles-ci peuvent se construire indépendamment d'elle, on doit admettre indirectement leur universalité, et du même coup on tombe dans l'illusion de notions pures qui peuvent exister indépendamment des langues particulières. Si, au contraire, on accepte l'idée d'une influence quelconque de la langue sur la formation des notions, on sera obligé de revoir la distinction entre notion et signifié.

Il est difficile d'admettre que le signifié d'un lexème (son sens déterminé dans le système linguistique) n'a aucun rapport avec sa signification une fois devenu terme, c'est-à-dire dès qu'il devient l'expression d'une notion déterminée dans les contours d'un groupe et d'une pratique sociale donnée.

C'est dans la mesure où des résidus sémantiques appartenant à un lexème restent collés à lui quand il devient terme qu'on doit parler de continuité sémantique entre signifié et notion. Maintenir une distinction catégorique entre les deux en considérant que la valeur du signifié dépend exclusivement du système de la langue et que celle des notions dépend exclusivement des pratiques sociales, c'est supposer que les lexèmes de la langue sont susceptibles d'être débarrassés de tout sémantisme en rapport avec leur valeur dans le système quand il arrive qu'ils soient réutilisés dans une pratique spécialisée donnée. Peut-on vraiment prétendre qu'entre les signifiés des lexèmes: agoniste, compatibilité, duplication et répression, pour ne citer que ceux-là, et les notions désignées par ces mêmes lexèmes (devenus termes dans la sphère d'activité des biotechnologies), il n'y aurait aucune continuité sémantique? Pour notre part, nous pensons qu'une partie des signifiés linguistiques de ces lexèmes est passée dans ces notions, comme on peut le constater à travers les énoncés définitoires suivants ( $c f$. Chopplet 1987):

agoniste: «Molécule qui mime l'effet principal d'une autre et donc entre en compétition avec elle»;

compatibilité : «En génie génétique, se dit de la capacité de deux plasmides de coexister de façon stable à l'intérieur d'un hôte»;

duplication: «Mécanisme de remaniement chromosomique par lequel un segment d'ADN va se transposer, c'est-à-dire se retrouver en deux copies séparées»; répression: «Blocage de la transcription d'un gène par les effecteurs protéiques appelés répresseurs».

Il serait difficile d'occulter la continuité sémantique entre le sens de ces lexèmes (sens entendu comme valeur linguistique déterminée dans le système) et leur signification en tant que termes réutilisés dans une activité spécialisée.

Dans ces divers cas, qui sont des exemples parmi beaucoup d'autres, un lecteur au fait des bases des biotechnologies, mais ignorant du terme agoniste, compatibilité, etc., se fera une idée du mécanisme ou de l'agent évoqué à partir de sa connaissance du vocabulaire général qu'il appliquera aux faits sur lesquels il s'informe. La notion naitra clairement pour lui, en ce cas, du transfert qu'il opérera.

À ce stade de notre analyse du rapport entre le signifié et la notion, nous pouvons légitimement considérer que le signifié d'un signe linguistique n'est rien d'autre que son sens déterminé dans et par le système linguistique, alors que la notion est la signification 
du terme déterminée par des sujets sociaux agissant au sein d'une activité spécialisée. Entre le sens et la signification, la frontière est perméable, c'est-à-dire qu'il y a une continuité qui peut être apparente ou latente selon les cas, mais qui existe. Cette continuité peut aller du sens vers la signification - c'est ce qui arrive, par exemple, quand un lexème devient terme - comme elle peut aller de la signification vers le sens - c'est ce qui arrive quand un terme passe dans le vocabulaire général.

Ce sur quoi il faut insister, c'est la dimension sociale de la signification. La part des sujets, comme acteurs sociaux concernés ou agissant directement dans le cadre d'une activité spécialisée, est importante dans la construction des significations. C'est dans les interactions verbales à l'occasion des préoccupations quotidiennes que les significations se dessinent et se fixent. «L'élaboration des idées, leur explication, leur mise à l'épreuve s'entrelacent en permanence au gré des multiples interactions qui saisissent les chercheurs, leurs commanditaires et leurs publics potentiels», nous dit avec raison Michel Callon (1988 : 176). Le laboratoire, à cet égard, ajoute cet auteur, occupe une position centrale : «non seulement c'est en son sein que s'observe la construction des interprétations ou des énoncés, mais c'est également là où se prépare et se gère la transformation de connaissances d'abord locales en connaissances négociées et échangées sur des marchés plus larges».

La dynamique sociale sous-jacente à la fabrication des significations est un fait incontestable notamment dans des secteurs d'activités, tels que les sciences et les technologies du vivant, où les contraintes économiques, sociologiques et culturelles ne sont pas sans importance. L'activité cognitive dans ce type de secteurs n'est pas sans aucun rapport avec les réalités de l'environnement extérieur, comme c'est le cas en logique formelle ou dans certains champs des mathématiques théoriques où l'activité cognitive et les concepts qui en découlent restent largement indépendants des réalités du monde extérieur.

Outre l'impact de la dimension sociale, la signification est conditionnée en partie aussi par les représentations que se font les sujets de la référence et qui sont partiellement fixées à partir des présupposés théoriques et de l'état des connaissances dans tel ou tel champ d'activités ( $c f$. Putnam 1990).

Du fait que ces connaissances soient évolutives, le rapport entre la notion et son référent est loin d'être une association stable : elle est susceptible de changer selon l'évolution des connaissances. Par conséquent, la notion ne peut contenir que les informations référentielles que les sujets connaissent ou qu'ils sont en mesure de connaître à un moment donné de l'histoire de leur pratique. Autrement dit, la notion peut contenir suffisamment d'informations pour une catégorisation de la référence, relative à l'état des connaissances, mais jamais assez d'informations pour l'explorer et l'identifier complètement. C'est en ce sens qu'il faut entendre le conditionnement de la signification par la référence, ou plus précisément par les représentations que les sujets se font d'elle.

Dire que les notions sont des représentations mentales des caractères objectifs inhérents aux objets et qu'elles sont indépendantes des langues individuelles (comme le veut la définition de l'ISO), c'est supposer, d'une part, qu'elles peuvent se construire par l'intermédiaire d'un langage autre que linguistique et que, d'autre part, elles n'ont pas besoin d'être énoncées en mots; mais y a-t-il un monde qui soit complètement indépendant des langues humaines?

Le mentalisme sous-jacent aux définitions normalisées de la notion en terminologie aboutit inévitablement à une dé-socialisation des notions pour en faire à tort des entités «psychologiques» où ne sont impliqués ni le langage, ni les enjeux sociaux de la connaissance. C'est cette attitude encore répandue qui stérilise la discipline terminologique en l'empêchant d'appréhender les termes et les notions dans ce qui constitue leur vraie identité, à savoir le fait d'être des unités de pensée linguistiquement et socialement 
déterminées, destinées à communiquer et à faire naître des savoirs dans les différents secteurs de la praxis humaine.

\section{RÉFÉRENCES}

CALLON, Michel (1988): La science et ses réseaux. Genèse et circulation des faits scientifiques, Paris, La découverte / Conseil de l'Europe.

CHOPPLET, Marc et al. (1987): Vocabulaire des biotechnologies, Paris, CESTA (Centre d'Études des Systèmes et des Technologies Avancées).

Dictionnaire des néologismes officiels (1990): «Arrêté du 14 septembre 1990 relatif à la terminologie du génie génétique», Paris, Journal Officiel.

FELBER, Helmut (1987) : Manuel de terminologie, Paris, UNESCO/Infoterm.

GARDN, Bernard (1988) : «Le sens comme production sociale», LINX, ${ }^{\circ}$ 19, Université de Paris X, pp. 43-54.

GAUDIN, F. (1990): Terminologie : des problèmes sémantiques aux pratiques institutionnelles, thèse de doctorat, Université de Rouen.

Norme internationale ISO 1087 (E/F): Terminologie-Vocabulaire, (1990),

PUTNAM, Hilary (1990) : Représentation et réalité, Paris, Gallimard.

REY, Alain (1979): La terminologie, noms et notions, Paris, PUF, coll. «Que sais-je?», $\mathrm{n}^{\circ} 1780$.

STENGERS, I. et J. SCHLANGER (1989): Les concepts scientifiques. Inventions et pouvoirs, Paris, La découverte / Conseil de l'Europe / UNESCO.

TAMBA-MECZ, Irène (1988) : La sémantique, Paris, PUF, coll. «Que sais-je ?», $\mathrm{n}^{\circ} 655$. 